\title{
Fibrinogen Level Predicts Outcomes in Critically Ill Patients with Acute Exacerbation of Chronic Heart Failure
}

\author{
Zhongyuan Meng $\mathbb{D}^{1}{ }^{1}$ Yaxin Zhao, ${ }^{1}$ and Yan $H e \mathbb{D}^{2}$ \\ ${ }^{1}$ Guangxi Medical University, Nanning, Guangxi, China \\ ${ }^{2}$ The First Affiliated Hospital of Guangxi Medical University, Nanning, Guangxi, China \\ Correspondence should be addressed to Yan He; hyxjwxy@126.com
}

Received 21 December 2020; Revised 3 April 2021; Accepted 9 April 2021; Published 30 April 2021

Academic Editor: Cecilia Vecoli

Copyright (c) 2021 Zhongyuan Meng et al. This is an open access article distributed under the Creative Commons Attribution License, which permits unrestricted use, distribution, and reproduction in any medium, provided the original work is properly cited.

\begin{abstract}
Background. Heart failure (HF) is a common cardiovascular disease, which is related to systemic inflammation for decades. Fibrinogen (FIB) is a sign of thrombosis and inflammation, which is associated with the prognosis of many diseases. Nevertheless, the role of fibrinogen level in the prognosis of critically ill patients with acute exacerbation of chronic heart failure is unclear. Methods. The data are from the Medical Information Mart for Intensive Care III (MIMIC III) database, which is a freely accessible critical care database. The primary outcome in our study was 90-day mortality. The prognostic value of fibrinogen was analyzed with receiver operating characteristic (ROC) curve analysis, Kaplan-Meier curve, and Cox model. Results. A total of 554 patients were included. Patients were divided into two groups, low fibrinogen level $(<284 \mathrm{mg} / \mathrm{dl})$ and high fibrinogen level $(\geq 284 \mathrm{mg} / \mathrm{dl})$, through the cut-off value of the ROC curve. The area under the ROC curve of fibrinogen for predicting 90-day mortality was 0.65 (95\% CI: 0.59-0.70). In the unadjusted Cox model, compared with the low fibrinogen level $(<284 \mathrm{mg} / \mathrm{dl})$, the 90 -day mortality of the hazard ratio (HR) with $95 \%$ confidence intervals (CI) of the high fibrinogen level is 3.33 (95\% CI 2.15-5.15). In different multivariable Cox models, compared with the low fibrinogen level ( $<284 \mathrm{mg} / \mathrm{dl})$, the 90 -day mortality of the hazard ratio of the high fibrinogen level is from 2.83 to 3.13. In subgroup analyses, significant interactions were observed only in age, chronic kidney disease (CKD), and APS III scores. Conclusion. Our data suggest that high fibrinogen levels $(\geq 284 \mathrm{mg} / \mathrm{dl}$ ) independently predict mortality in critically ill patients with acute exacerbation of chronic heart failure. Our findings need to be further validated by large prospective studies and longer follow-up time.
\end{abstract}

\section{Introduction}

Heart failure (HF) is a common cardiovascular disease, and the prevalence of heart failure has increased to 6.2 million in Americans over the age of 20 [1]. Heart failure is a disorder of the myocardial structure and function, which leads to restricted ventricular ejection or filling, impaired blood circulation throughout the body, and insufficient tissue and organ perfusion [2]. Heart failure has been seen to be related to systemic inflammation for decades $[3,4]$.

Fibrinogen (FIB) is produced by the liver and activated during the clotting process [5], which is a sign of thrombosis and inflammation [6]. In addition, fibrinogen is also related to the prognosis of many diseases, including coronary artery disease [7-9], diabetes $[10,11]$, and chronic kidney disease
[12]. Fibrinogen levels were increased in patients with chronic cardiac insufficiency [13-15]. Nevertheless, most previous studies only analyzed how heart failure affects fibrinogen levels [16-18].

The influence of relative fibrinogen level on the prognosis of patients with acute exacerbation of chronic heart failure still lacks relevant research. Therefore, we intend to investigate the effect of the fibrinogen level on the prognosis of acute exacerbation of chronic heart failure. A better understanding of the relationship between fibrinogen level and acute exacerbation of chronic heart failure may help in risk stratification and assist in treatment to reduce mortality. The purpose of our study was to explore the effect of fibrinogen level on the prognosis of severe patients with acute exacerbation of chronic heart failure. 


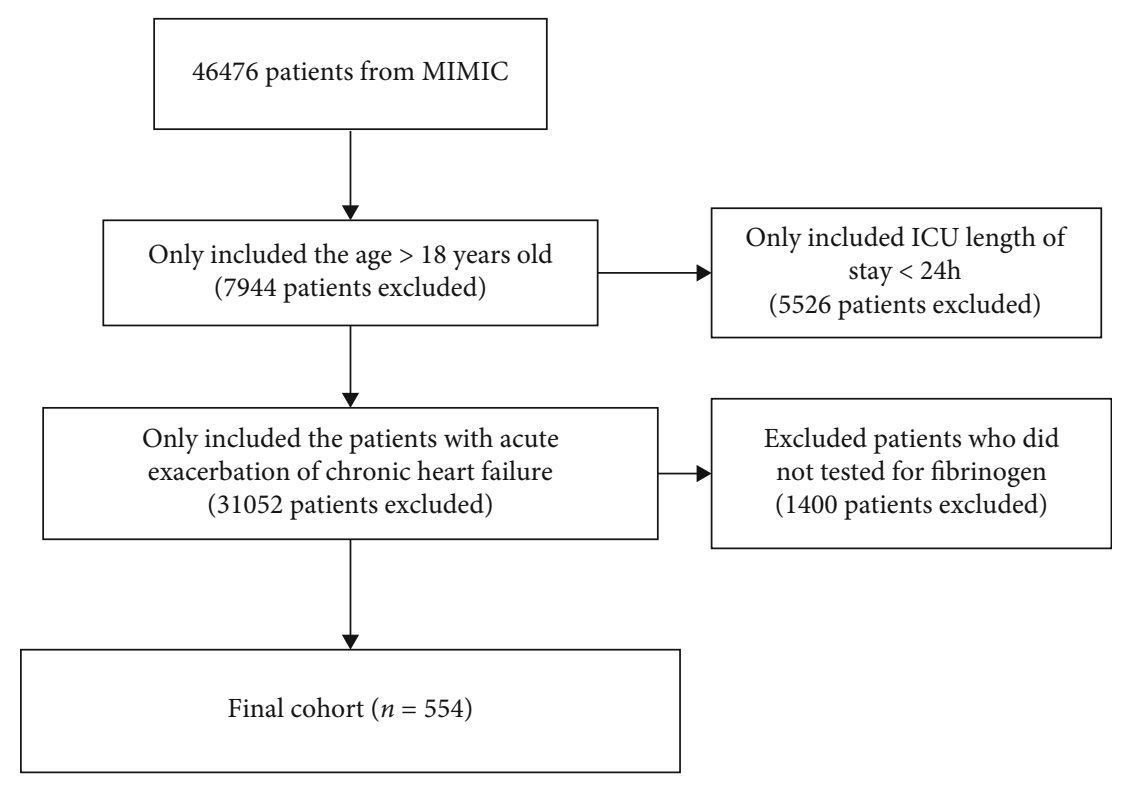

FIgure 1: The flowchart of patient selection.

\section{Methods}

The data for all the studies are from the Medical Information Mart for Intensive Care III (MIMIC III) database, which is a freely accessible critical care database [19]. MIMIC III comprised about 60000 ICU admissions at the Beth Israel Deaconess Medical Center in Boston. All subjects' names were replaced by subject_id, which fully protects the patient's privacy. The information recorded in the database includes demographic characteristics, diagnosis, laboratory examination data, microbial culture data, imaging data, nursing records, treatment measures, and fluid balance. After completing the National Institutes of Health web-based training course, we are eligible to access the database. Data were extracted by author Meng who has completed an online training course at the National Institutes of Health (Zhongyuan Meng, certification number: 9071533).

2.1. Subjects Selection. We selected all patients in the database with the first ICU admission. The inclusion criteria were the following: (1) the patients were older than 18 years old, (2) the patients stayed in ICU more than 24 hours, (3) the patients had acute exacerbation of chronic heart failure, and (4) the patients had data on the fibrinogen.

2.2. Data Extraction. Data extracted from the MIMIC III database includes gender, age, ethnicity, BMI, mean blood pressure, sequential organ failure assessment (SOFA) score, Acute Physiology Score III (APS III score), Systemic Inflammatory Response Syndrome (SIRS score), Simplified Acute Physiology Score II (SAPS II score), vasopressor use, ventilator use, date of hospital admission and discharge, date of ICU admission and discharge, and date of birth and death. Comorbidities included atrial fibrillation (Af), high blood pressure (HBP), diabetes mellitus (DM), chronic kidney disease $(\mathrm{CKD})$, acute kidney injury (AKI), respiratory failure, and acute myocardial infarction (AMI). Laboratory examination includes hemoglobin (Hb), platelet (PLT) count, white blood cell (WBC) count, fibrinogen, serum creatine (Scr), NT-proBNP, and serum glucose. All data were the results of the patient's initial examination after hospital admission. As there are too many missing records of NTproBNP, we converted it into a dummy variable in the model. The primary outcome in our study was 90-day mortality, defined as death occurring within 90 days after admission. In addition, we made a preliminary assessment of 28day mortality and ICU length of stay (LOS).

2.3. Statistical Analyses. The measurement data consistent with normal distribution were expressed as mean \pm standard deviation, and the comparison between groups was performed by the $t$-test. Nonnormally distributed data were represented by median and interquartile difference (IQR), and the Wilcoxon rank sum test was used for comparison between groups. The categorical variables are presented by frequency and used by the chi-square test. The receiver operating characteristic (ROC) curve analysis, Kaplan-Meier curve, and Cox proportional hazards models were performed to determine the association between fibrinogen and 90-day mortality. ROC results showed that the cutoff value of fibrinogen predicting 90-day mortality was $284 \mathrm{mg} / \mathrm{dl}$. Thus, the study population was divided into two groups, namely, low fibrinogen group $(<284 \mathrm{mg} / \mathrm{dl}$ group) and high fibrinogen group $(\geq 284 \mathrm{mg} / \mathrm{dl}$ group) according to fibrinogen. The low fibrinogen level was selected as the reference group. To control the influence of confounders, we choose multivariable analysis to adjust. Multicollinearity was tested by a variance inflation factor (VIF). VIF $\geq 5$ indicated the existence of multicollinearity. 
TABLE 1: Summary of patient characteristics.

\begin{tabular}{|c|c|c|c|}
\hline & Total $(n=554)$ & Fibrinogen level < 284 mg/dl $(n=225)$ & Fibrinogen level $\geq 284 \mathrm{mg} / \mathrm{dl}(n=329)$ \\
\hline Age (years) & $72.2 \pm 13.7$ & $71.4 \pm 12.8$ & $72.7 \pm 14.2$ \\
\hline BMI $\left(\mathrm{kg} / \mathrm{m}^{2}\right)$ & $28.7 \pm 6.4$ & $28.1 \pm 5.8$ & $29.1 \pm 6.7$ \\
\hline Gender (male), $n(\%)$ & $319(57.6)$ & $124(55.1)$ & $195(59.3)$ \\
\hline \multicolumn{4}{|l|}{ Ethnicity, $n(\%)$} \\
\hline White & $426(76.9)$ & $174(77.3)$ & $252(76.6)$ \\
\hline Black & $33(6.0)$ & $12(5.3)$ & $21(6.4)$ \\
\hline Other & $95(17.1)$ & $39(17.3)$ & $56(17.0)$ \\
\hline Mean BP (mmHg) & $73.4 \pm 9.1$ & $72.7 \pm 7.7$ & $73.8 \pm 10.0$ \\
\hline \multicolumn{4}{|l|}{ Laboratory outcomes } \\
\hline WBC count $\left(10^{9} / 1\right)$ & $13.1 \pm 6.6$ & $13.3 \pm 5.9$ & $12.9 \pm 7.1$ \\
\hline $\mathrm{Hb}(\mathrm{mg} / \mathrm{dl})$ & $10.0 \pm 2.2$ & $9.8 \pm 2.5$ & $10.2 \pm 2.0$ \\
\hline $\mathrm{Scr}(\mathrm{mg} / \mathrm{dl})$ & $1.5 \pm 1.1$ & $1.2 \pm 0.7$ & $1.7 \pm 1.3$ \\
\hline NT-ProBNP, $n(\%)$ & $70(12.6)$ & $12(5.3)$ & $58(17.6)$ \\
\hline Platelet count $\left(10^{9} / 1\right)$ & $195.9 \pm 100.8$ & $164.7 \pm 74.1$ & $217.2 \pm 110.8$ \\
\hline Glucose (mg/dl) & $152.8 \pm 66.3$ & $150.1 \pm 63.7$ & $154.7 \pm 68.1$ \\
\hline Fibrinogen (mg/dl) & $371.1 \pm 198.3$ & $200.4 \pm 53.1$ & $487.8 \pm 175.3$ \\
\hline \multicolumn{4}{|l|}{ Comorbidities, $n$ (\%) } \\
\hline Respiratory failure, $n(\%)$ & $185(33.4)$ & $46(20.4)$ & $139(42.2)$ \\
\hline $\mathrm{HBP}, n(\%)$ & $265(47.8)$ & $121(53.8)$ & $144(43.8)$ \\
\hline $\mathrm{DM}, n(\%)$ & $224(40.4)$ & $84(37.3)$ & $140(42.6)$ \\
\hline Af, $n(\%)$ & $291(52.5)$ & $123(54.7)$ & $168(51.1)$ \\
\hline AKI, $n(\%)$ & $512(92.4)$ & $210(93.3)$ & $302(91.8)$ \\
\hline $\mathrm{CKD}, n(\%)$ & $273(49.3)$ & $89(39.6)$ & $184(55.9)$ \\
\hline NSTEMI, $n(\%)$ & $131(13.6)$ & $47(20.9)$ & $84(25.5)$ \\
\hline STEMI, $n(\%)$ & $32(5.8)$ & $7(3.1)$ & $25(7.6)$ \\
\hline Vasopressor use, $n(\%)$ & $403(72.7)$ & $177(78.7)$ & $226(68.7)$ \\
\hline Ventilator use, $n(\%)$ & $427(77.1)$ & $192(85.3)$ & $235(71.4)$ \\
\hline CRRT & $41(7.4)$ & $11(4.9)$ & $30(9.1)$ \\
\hline \multicolumn{4}{|l|}{ Disease scores } \\
\hline SOFA scores & $6(4-9)$ & $4(7-9)$ & $6(4-8)$ \\
\hline APS III scores & $48(35-63)$ & $42(31-58)$ & $52(40-67)$ \\
\hline SAPS II scores & $42(35-52)$ & $40(34-51)$ & $44(35-53)$ \\
\hline SIRS scores & $3(2-4)$ & $3(2-3)$ & $3(3-4)$ \\
\hline \multicolumn{4}{|l|}{ Outcome } \\
\hline ICU LOS (days) & $4.9(2.7-8.6)$ & $3.3(2.5-6.1)$ & $6.1(3.2-10.2)$ \\
\hline 28-day mortality, $n(\%)$ & $82(14.8)$ & $15(6.7)$ & $67(20.4)$ \\
\hline 90-day mortality, $n(\%)$ & $133(24.0)$ & $25(11.1)$ & $108(32.8)$ \\
\hline
\end{tabular}

Model 1 was adjusted for only fibrinogen, PLT, NT-proBNP, vasopressor use, and ventilator use. Model $2=$ model 1 $+\mathrm{HBP}$, Af, respiratory failure, chronic kidney disease, and STEMI. Due to the multicollinearity in each severe disease score, only one score is added to each model for correction. Model $3=$ model $2+$ SIRS scores (VIF 4.24). Model $4=$ model 2+APS III scores (VIF 3.94). Model $5=$ model 2+SAPS II scores (VIF 4.22). Stata16 MP was used for statistical analysis of all data; a two-side $P<0.05$ was considered statistically significant.

\section{Result}

554 patients met the inclusion criteria. The flowchart of patient selection is shown in Figure 1. Table 1 lists the basic characteristics of all patients by the low fibrinogen group $(<284 \mathrm{mg} / \mathrm{dl}$ group) and high fibrinogen group $(\geq 284 \mathrm{mg} / \mathrm{dl}$ group). There were 225 patients in the low fibrinogen group and 329 patients in the high fibrinogen group. The high fibrinogen group tended to have high PLT count, high Scr, HBP, CKD, STEMI, vasopressor use, ventilator use, high 

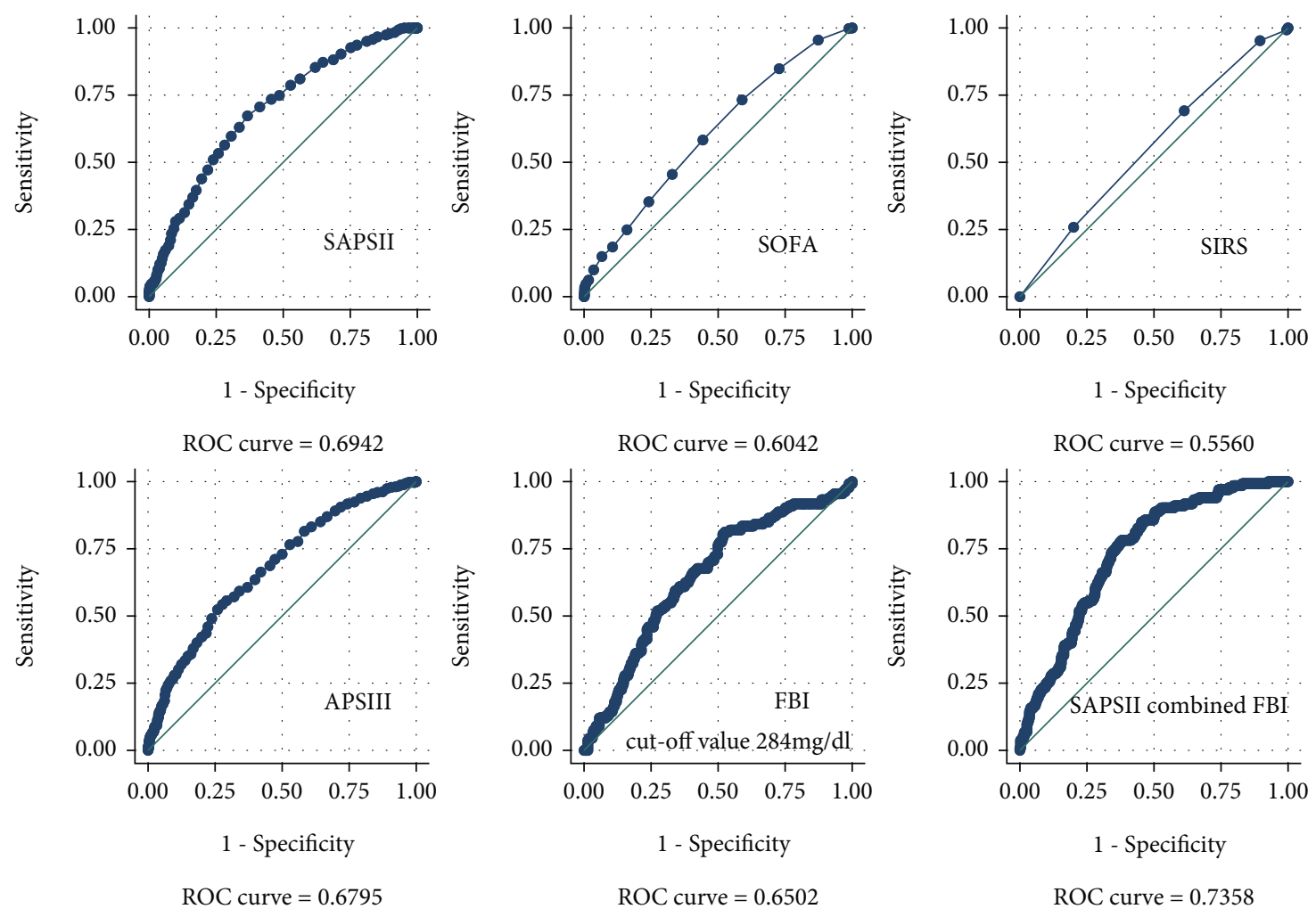

FIGURE 2: Receiver operating characteristic curves of fibrinogen and severity scores for predicting 90-day mortality.

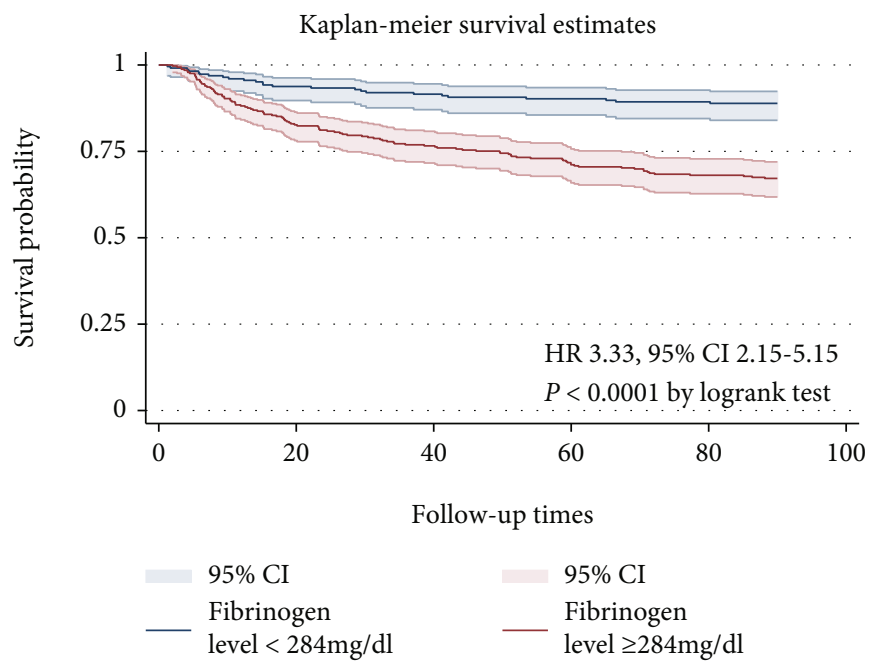

Figure 3: Kaplan-Meier curves for 90-day mortality.

APS III, SAPS II, and SIRS scores. Figure 2 shows the ROC curves of fibrinogen and different severity scores. In all ROC curves, the area of AUC is less than 0.7. However, after combining fibrinogen and SAPS II, the AUC of the joint prediction model reaches 0.73 (95\% CI 0.69-0.78), which is higher than that of fibrinogen and other disease severity scores alone.

Figure 3 shows the Kaplan-Meier curve for patients in the low and high fibrinogen groups. A higher fibrinogen was significantly associated with risk of 90-day mortality $(P<0.0001$ by the logrank test). In Table 2 , we use the Cox model to analyze the association between fibrinogen and 90-day mortality. In the unadjusted Cox model, compared with the low fibrinogen group, the 90-day mortality of $\mathrm{HR}(95 \% \mathrm{CI})$ of the high fibrinogen group is 3.33 (95\% CI, 2.15-5.15). In the extended multiple COX model, high fibrinogen levels were significantly associated with increased 90-day mortality, in model 1 (HR 3.35, 95\% CI 1.95-5.76), 
TABLE 2: Association between fibrinogen and 90-day mortality.

\begin{tabular}{lcccc}
\hline & $\begin{array}{c}\text { Fibrinogen } \\
\text { level }(\mathrm{mg} / \mathrm{dl})\end{array}$ & Hazard ratio & $95 \%$ CI & $P$ value \\
\hline \multirow{2}{*}{ Crude } & $<284$ & Ref & & \\
& $\geq 284$ & 3.33 & $2.15-5.15$ & $<0.0001$ \\
Model 1 & $<284$ & Ref & & \\
& $\geq 284$ & 3.35 & $1.95-5.76$ & $<0.0001$ \\
Model 2 & $<284$ & Ref & & \\
& $\geq 284$ & 3.11 & $1.81-5.36$ & $<0.0001$ \\
Model 3 & $<284$ & Ref & & \\
& $\geq 284$ & 3.13 & $1.82-5.40$ & $<0.0001$ \\
Model 4 & $<284$ & Ref & & \\
& $\geq 284$ & 2.83 & $1.63-4.90$ & $<0.0001$ \\
Model 5 & $<284$ & Ref & & \\
& $\geq 284$ & 2.85 & $1.65-4.92$ & $<0.0001$ \\
\hline
\end{tabular}

Adjusted covariates: model $1=$ fibrinogen, PLT, NT-proBNP, vasopressor use, and ventilator use; model $2=$ model $1+\mathrm{HBP}$, Af, respiratory failure, CKD, and STEMI; model $3=$ model 2+SIRS scores (VIF 4.24); model $4=$ model $2+$ APS III scores (VIF 3.94); model $5=$ model $2+$ SAPS II scores (VIF 4.22).

model 2 (HR 3.11, 95\% CI 1.81-5.36), model 3 (HR 3.13, 95\% CI 1.82-5.40), model 4 (HR 2.83, 95\% CI 1.63-4.90), and model 5 (HR 2.85, 95\% CI 1.65-4.92).

3.1. Subgroup Analysis. Subgroup analysis was used to assess association between fibrinogen and 90-day mortality in different groups (Table 3). Significant interactions were observed in age, CKD, and APS III scores. For patients $\geq 65$ years old, the risk of 90-day mortality was significantly higher in the high fibrinogen group (HR 4.16, 95\% CI 2.516.88), whereas we did not find this relationship in patients $<65$ years old (HR 1.29, 95\% CI 0.50-3.28). In patients without $\mathrm{CKD}$, the risk of 90 -day mortality was significantly higher in the high fibrinogen group (HR 9.87, 95\% 3.9124.8). Similarly, in the APS III scores $<48$ group, the risk of 90-day mortality was significantly higher in the high fibrinogen group (HR 7.69, 95\% 2.69-21.9).

\section{Discussion}

At present, there are few studies to report the effect of fibrinogen on the prognosis of acute exacerbation of chronic heart failure. In the current study, we explored the connection between fibrinogen levels and the prognosis of patients with acute exacerbation of chronic heart failure. In our study, we found that fibrinogen levels $\geq 284 \mathrm{mg} / \mathrm{dl}$ had a significant correlation with mortality. Compared to the prediction accuracy of fibrinogen with different severe disease scores, we observed that only SAPS II and APS III had a slightly higher predictive accuracy than fibrinogen. However, the acquisition of SAPS II and APS III requires measurement of multiple data, including not only general vital signs but also blood gas analysis results, etc. Therefore, these difficulties bring certain challenges to clinical use. On the contrary, fibrinogen is an easy-to-measure indicator, which is convenient for clinical use. Of course, we can also significantly improve the prediction accuracy when we combine fibrinogen and disease severity scores.

In contrast to our findings, Chin et al. [20] studied 120 outpatients with chronic stable heart failure and found that there was no significant correlation between fibrinogen levels and all-cause mortality. There are several factors that may contribute to the inconsistencies in the results. We studied critically ill patients with acute episodes of chronic heart failure, while Chin et al. studied patients with chronic stable heart failure. Outcomes between different cohorts may also vary greatly. Our cohort study revealed that patients with acute episodes of chronic heart failure at a higher level of fibrinogen (threshold value $>284 \mathrm{mg} / \mathrm{dl}$ ) had a higher risk of 90-day mortality compared to patients with a lower level. In our study, after adjusting the related covariates, high fibrinogen levels still showed a strong correlation with 90day mortality (HR from 2.83 to $3.13, P<0.0001$ ).

There is increasing evidence that fibrinogen is a poor prognostic predictor of cardiovascular disease. Fibrinogen may increase cardiovascular risk through platelet aggregation, plasma viscosity, and fibrin formation [21]. Stec et al. [22] measured fibrinogen levels in 2,632 subjects using a newly developed immunoprecipitation test; they suggest that fibrinogen is associated with traditional cardiovascular risk factors. A study by Kotbi et al. [23] has also shown that fibrinogen is related to the severity of coronary artery disease and cardiovascular risk in Moroccan patients. A large meta-analysis found a moderately strong correlation between plasma fibrinogen levels and coronary heart disease risk [24]. In addition, Song et al. [6] suggested that fibrinogen was an independent predictor of reinfarction after PCI in NSTE-ACS patients. After adjusting for variates, FIB level also is associated with a higher rate of death/nonfatal reinfarction $(\mathrm{HR}=1.498,95 \% \mathrm{CI}: 1.030$ 2.181, $P=0.035)$. Although these studies are different, most of the conclusions reached are very similar to our research; that is, fibrinogen is closely related to the prognosis of cardiovascular disease.

We studied the possibility of the relationship between fibrinogen and mortality in patients with acute exacerbation of chronic heart failure. Fibrinogen is easy to obtain and convenient for clinical use. Admission fibrinogen measurement may be used to stratify the prognosis risk of acute exacerbation of chronic heart failure and provide a reference for later treatment. To our knowledge, this seems to be the first study to evaluate the relationship between fibrinogen and the prognosis of acute exacerbations of chronic cardiac function, and this may be helpful for clinical practice.

4.1. Study Limitation. Our study had several limitations. Although we found that high fibrinogen is independently associated with adverse outcomes, our study is still a single-center retrospective study. We only collected the data of the patient on admission. The relationship between the dynamic changes of fibrinogen and patients with acute exacerbation of chronic heart failure cannot be analyzed. 
TABLE 3: Subgroup analysis of the associations between fibrinogen and 90-day mortality.

\begin{tabular}{|c|c|c|c|c|}
\hline Subgroup & $N$ & $\begin{array}{l}\mathrm{HR}(95 \% \mathrm{CI}) \\
<284 \mathrm{mg} / \mathrm{dl}\end{array}$ & $\begin{array}{l}\mathrm{HR}(95 \% \mathrm{CI}) \\
\geq 284 \mathrm{mg} / \mathrm{dl}\end{array}$ & $\begin{array}{c}P \text { for } \\
\text { interaction }\end{array}$ \\
\hline Gender & & & & 0.198 \\
\hline Male & 235 & Ref & $4.70(2.30-9.57)$ & \\
\hline Female & 319 & & $2.60(1.50-4.53)$ & \\
\hline Age & & & & 0.030 \\
\hline$<65$ & 149 & Ref & $1.29(0.50-3.28)$ & \\
\hline$\geq 65$ & 405 & & $4.16(2.51-6.88)$ & \\
\hline HBP & & & & 0.066 \\
\hline Yes & 265 & Ref & $5.42(2.55-11.51)$ & \\
\hline No & 289 & & $2.29(1.34-3.91)$ & \\
\hline $\mathrm{DM}$ & & & & 0.116 \\
\hline Yes & 224 & Ref & $2.21(1.16-4.21)$ & \\
\hline No & 330 & & $4.46(2.46-8.09)$ & \\
\hline Af & & & & 0.388 \\
\hline Yes & 291 & Ref & $3.97(2.23-7.09)$ & \\
\hline No & 263 & & $2.68(1.38-5.20)$ & \\
\hline $\begin{array}{l}\text { Respiratory } \\
\text { failure }\end{array}$ & & & & 0.346 \\
\hline Yes & 185 & Ref & $2.16(1.14-4.11)$ & \\
\hline No & 369 & & $3.28(1.80-5.97)$ & \\
\hline CKD & & & & 0.001 \\
\hline Yes & 273 & Ref & $1.61(0.97-2.67)$ & \\
\hline No & 281 & & $9.87(3.91-24.8)$ & \\
\hline \multicolumn{5}{|l|}{ AKI } \\
\hline Yes & 512 & Ref & $3.36(2.17-5.20)$ & \\
\hline No & 42 & & NA & \\
\hline NSTEMI & & & & 0.992 \\
\hline Yes & 131 & Ref & $3.31(1.27-8.63)$ & \\
\hline No & 423 & & $3.35(2.05-5.46)$ & \\
\hline STEMI & & & & 0.161 \\
\hline Yes & 32 & Ref & $1.12(0.23-5.30)$ & \\
\hline No & 522 & & $3.53(2.24-5.56)$ & \\
\hline $\begin{array}{l}\text { Vasopressor } \\
\text { use }\end{array}$ & & & & 0.304 \\
\hline Yes & 403 & Ref & $3.81(2.31-6.30)$ & \\
\hline No & 151 & & $2.29(0.94-5.54)$ & \\
\hline Ventilator use & & & & 0.085 \\
\hline Yes & 427 & Ref & $4.00(2.43-6.60)$ & \\
\hline No & 127 & & $1.63(0.67-3.94)$ & \\
\hline CRRT use & & & & 0.111 \\
\hline Yes & 41 & Ref & $1.59(0.64-3.96)$ & \\
\hline No & 513 & & $3.63(2.21-5.98)$ & \\
\hline SOFA scores & & & & 0.293 \\
\hline$<6$ & 234 & Ref & $4.95(2.11-11.60)$ & \\
\hline$\geq 6$ & 320 & & $2.86(1.71-4.79)$ & \\
\hline APS III scores & & & & 0.023 \\
\hline$<48$ & 271 & Ref & $7.69(2.69-21.9)$ & \\
\hline$\geq 48$ & 283 & & $1.96(1.21-3.17)$ & \\
\hline
\end{tabular}

TABle 3: Continued.

\begin{tabular}{lcccc}
\hline Subgroup & $N$ & $\begin{array}{c}\text { HR }(95 \% \mathrm{CI}) \\
<284 \mathrm{mg} / \mathrm{dl}\end{array}$ & $\begin{array}{c}\text { HR }(95 \% \mathrm{CI}) \\
\geq 284 \mathrm{mg} / \mathrm{dl}\end{array}$ & $\begin{array}{c}P \text { for } \\
\text { interaction }\end{array}$ \\
\hline $\begin{array}{l}\text { SAPS II scores } \\
\quad\end{array}$ & & & 0.966 \\
$\quad<42$ & 271 & Ref & $3.16(1.44-6.94)$ & \\
$\quad \geq 42$ & 283 & & $3.05(1.80-5.15)$ & \\
SIRS scores & & & & 0.845 \\
$\quad<3$ & 150 & Ref & $3.57(1.45-8.78)$ & \\
$\geq 3$ & 404 & & $3.20(1.94-5.26)$ & \\
\hline
\end{tabular}

\section{Conclusion}

Our data suggest that high fibrinogen levels $(\geq 284 \mathrm{mg} / \mathrm{dl})$ independently predict mortality in critically ill patients with acute exacerbation of chronic heart failure. Our findings need to be further validated by large prospective studies and longer follow-up time.

\section{Abbreviation}

HF: $\quad$ Heart failure

BMI: Body mass index

Af: $\quad$ Atrial fibrillation

AMI: Acute myocardial infarction

Scr: $\quad$ Serum creatine

LOS: Length of stay

HR: Hazard ratio

CI: $\quad 95 \%$ confidence intervals

SOFA: Sequential organ failure assessment

APS III: Acute Physiology Score III

SIRS: $\quad$ Systemic Inflammatory Response Syndrome

SAPS II: Simplified Acute Physiology Score II

DM: Diabetes mellitus

CKD: Chronic kidney disease

AKI: Acute kidney injury

Hb: Hemoglobin

PLT: $\quad$ Platelets

WBC: White blood cells

FIB: $\quad$ Fibrinogen.

\section{Data Availability}

The data used in the study is available at https://mimic .physionet.org/.

\section{Conflicts of Interest}

The authors declare that they have no conflicts of interest.

\section{References}

[1] S. S. Virani, A. Alonso, E. J. Benjamin et al., "Heart disease and stroke statistics-2020 update: a report from the American Heart Association," Circulation, vol. 141, no. 9, pp. e139-e596, 2020.

[2] Y. Tang, W. Lin, L. Zha et al., "Serum anion gap is associated with all-cause mortality among critically ill patients with congestive heart failure," Disease Markers, vol. 2020, Article ID 8833637, 10 pages, 2020. 
[3] S. P. Murphy, R. Kakkar, C. P. McCarthy, and J. L. Januzzi Jr., "Inflammation in heart failure:," Journal of the American College of Cardiology, vol. 75, no. 11, pp. 1324-1340, 2020.

[4] L. Adamo, C. Rocha-Resende, S. D. Prabhu, and D. L. Mann, "Reappraising the role of inflammation in heart failure," Nature Reviews. Cardiology, vol. 17, no. 5, pp. 269-285, 2020.

[5] I. Induruwa, M. Moroi, A. Bonna et al., "Platelet collagen receptor glycoprotein VI-dimer recognizes fibrinogen and fibrin through their D-domains, contributing to platelet adhesion and activation during thrombus formation," Journal of Thrombosis and Haemostasis, vol. 16, no. 2, pp. 389-404, 2018.

[6] J. Song, T. T. Yu, Z. J. Sun, Z. C. Li, D. X. He, and Z. Q. Sun, "Comparison of prognostic significance between serum fibrinogen and Global Registry of Acute Coronary Events score for prognosis of patients with non-ST-elevation acute coronary syndromes undergoing percutaneous coronary intervention," Coronary Artery Disease, vol. 31, no. 2, pp. 124-129, 2020.

[7] Ø. R. Mjelva, G. F. T. Svingen, E. K. R. Pedersen et al., "Fibrinogen and neopterin is associated with future myocardial infarction and total mortality in patients with stable coronary artery disease," Thrombosis and Haemostasis, vol. 118, no. 4, pp. 778-790, 2018.

[8] S. L. Liu, N. Q. Wu, H. W. Shi et al., "Fibrinogen is associated with glucose metabolism and cardiovascular outcomes in patients with coronary artery disease," Cardiovascular Diabetology, vol. 19, no. 1, p. 36, 2020.

[9] G. Coppola, M. Rizzo, M. G. Abrignani et al., "Fibrinogen as a predictor of mortality after acute myocardial infarction: a forty-two-month follow-up study," Italian Heart Journal, vol. 6, no. 4, pp. 315-322, 2005.

[10] S. A. Gracheva, M. S. Biragova, A. M. Glazunova et al., "Risk factors for and prognostic value of multifocal atherosclerosis in patients with diabetes mellitus," Terapevticheskiu Arkhiv, vol. 86, no. 10, pp. 20-26, 2014.

[11] S. H. Yang, Y. du, Y. Zhang et al., "Serum fibrinogen and cardiovascular events in Chinese patients with type 2 diabetes and stable coronary artery disease: a prospective observational study," BMJ Open, vol. 7, no. 6, article e015041, 2017.

[12] M. Goicoechea, S. G. de Vinuesa, F. Gomez-Campdera et al., "Serum fibrinogen levels are an independent predictor of mortality in patients with chronic kidney disease (CKD) stages 3 and 4," Kidney International. Supplement, vol. 111, pp. S67S70, 2008.

[13] A. Mongirdiene, L. Kursvietiene, and A. Kasauskas, "The coagulation system changes in patients with chronic heart failure," Medicina (Kaunas, Lithuania), vol. 46, no. 9, pp. 642-647, 2010.

[14] K. K. Witte, S. J. Ford, T. Preston, J. D. Parker, and A. L. Clark, "Fibrinogen synthesis is increased in cachectic patients with chronic heart failure," International Journal of Cardiology, vol. 129, no. 3, pp. 363-367, 2008.

[15] T. J. Zhao, Q. K. Yang, C. Y. Tan, L. D. Bi, J. Li, and Z. L. Miao, "Prognostic value of D-dimer/fibrinogen ratio in the adverse outcomes of patients hospitalized for heart failure," Biomarkers in Medicine, vol. 14, no. 18, pp. 1733-1745, 2020.

[16] A. Hoffmeister, J. Hetzel, S. Sander, M. Kron, V. Hombach, and W. Koenig, "Plasma viscosity and fibrinogen in relation to haemodynamic findings in chronic congestive heart failure," European Journal of Heart Failure, vol. 1, no. 3, pp. 293-295, 1999.
[17] G. Engstrom, B. Hedblad, P. Tyden, and F. Lindgarde, "Inflammation-sensitive plasma proteins are associated with increased incidence of heart failure: a population-based cohort study," Atherosclerosis, vol. 202, no. 2, pp. 617-622, 2009.

[18] I. Palka, J. Nessler, B. Nessler, W. Piwowarska, W. Tracz, and A. Undas, "Altered fibrin clot properties in patients with chronic heart failure and sinus rhythm: a novel prothrombotic mechanism," Heart, vol. 96, no. 14, pp. 1114-1118, 2010.

[19] A. E. W. Johnson, T. J. Pollard, L. Shen et al., "MIMIC-III, a freely accessible critical care database," Scientific Data, vol. 3, no. 1, 2016.

[20] B. S. Chin, A. D. Blann, C. R. Gibbs, N. A. Chung, D. G. Conway, and G. Y. Lip, "Prognostic value of interleukin-6, plasma viscosity, fibrinogen, von Willebrand factor, tissue factor and vascular endothelial growth factor levels in congestive heart failure," European Journal of Clinical Investigation, vol. 33, no. 11, pp. 941-948, 2003.

[21] P. Jiang, Z. Gao, W. Zhao et al., "Relationship between fibrinogen levels and cardiovascular events in patients receiving percutaneous coronary intervention: a large single-center study," Chinese Medical Journal, vol. 132, no. 8, pp. 914-921, 2019.

[22] J. J. Stec, H. Silbershatz, G. H. Tofler et al., "Association of fibrinogen with cardiovascular risk factors and cardiovascular disease in the Framingham Offspring Population," Circulation, vol. 102, no. 14, pp. 1634-1638, 2000.

[23] S. Kotbi, A. Mjabber, A. Chadli et al., "Relation entre taux plasmatiques de fibrinogene et severite de l'atteinte coronarienne chez les patients diabetiques de type 2," Prospective study. Ann Endocrinol-Paris, vol. 77, no. 5, pp. 606-614, 2016.

[24] Fibrinogen Studies Collaboration, J. Danesh, S. Lewington et al., "Plasma fibrinogen level and the risk of major cardiovascular diseases and nonvascular mortality - an individual participant meta-analysis," JAMA, vol. 294, no. 14, pp. 17991809, 2005. 